\title{
The core of the core: What is at the heart of hospital core midwifery practice in New Zealand?
}

\author{
Andrea Gilkison ${ }^{A, B}$ PhD, MEd(Dist), BA, RM, RCpN • Judith McAra-Couper ${ }^{B}$ PhD, BA, RM - Anna \\ Fielder ${ }^{B}$ MPhil, DPSM, PGDip, BA(Hons) - Marion Hunter ${ }^{B}$ DHSC Candidate, MA(Hons), BA, ADN, RM, \\ RGON • Diana Austin ${ }^{B}$ DHSc Candidate, MA(Hons), BA, RM, RCpN
}

${ }^{\mathrm{A}}$ Corresponding

Author: andrea.

gilkison@aut.ac.nz

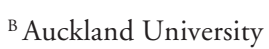
of Technology, New Zealand

\begin{abstract}
Background: New Zealand midwives who are employed by District Health Boards and are based in hospitals and maternity units are known as core midwives. Half of New Zealand midwives are employed as core midwives, performing a variety of key roles and, as such, are central to the functioning of maternity services. The sustainability of core midwifery is therefore highly significant for the future of maternity services in New Zealand. Research on sustainable midwifery practice operates as a constructive counterpoint to the growing literature on burnout and stress amongst midwives.
\end{abstract}

Aim: The question this study asked is: What sustains midwives who have been in hospital practice in New Zealand for more than eight years? The findings will inform workforce planners, managers and the midwifery profession about what may well contribute to the retention of midwives who are essential to the maternity services provided in hospital settings.

Methods: A qualitative descriptive study was conducted in New Zealand, recruiting and interviewing 22 core midwives with between 8 and 40-plus years' experience. Interviews were transcribed and thematic analysis was undertaken by the research team. Analysis was done as a group in a reciprocal fashion between the individual interviews and the data as a whole. Themes were clustered into groups and excerpts from the data used to illustrate the agreed themes. Ethical approval was obtained from Auckland University of Technology Ethics Committee.

Findings: This study found that core midwives sustain themselves in practice through developing significant core midwifery skills. Core midwives quickly build a partnership with women; and they are prepared to deal with everything, including unexpected and critical incidents. Core midwives often take on a managerial role in a unit and, as such, create the culture of the unit while supporting students and new graduates, as well as Lead Maternity Carers.

Conclusion: Core midwives highlight the importance of effective relationships with women, whānau, colleagues and managers. Our sample displayed unique and specific skills: connecting quickly with women, anticipating ahead to keep women safe, managing complexity, being prepared for everything, managing a unit and displaying flexibility and adaptability in their work. However, these core midwives feel invisible and undervalued at times, a finding that may well shine much needed light on what threatens sustainability of the core midwifery service nationwide.

Keywords: core midwifery skills, hospital core midwives, sustainable midwifery

\section{INTRODUCTION}

\section{Background and rationale for the study}

Research on sustainable midwifery operates as a constructive counterpoint to the growing literature on burnout and stress amongst midwives. Studies that have explored sustainable midwifery practice in New Zealand have tended to focus upon the experiences of caseloading Lead Maternity Carer (LMC) midwives (Engel, 2003; Gilkison et al., 2015; McAra-Couper et al., 2014; Wakelin \& Skinner, 2007). Of the midwives who held a current midwifery annual practising certificate in October 2015, 50.2\% reported core practice as their main work (Midwifery Council of New Zealand, 2015). This study explores the experiences of a group of such midwives. In this study the term "core midwife" has been used to refer to all midwives who are employed (usually by District Health Boards, DHBs) and are based in hospitals or maternity units, or who have other employed roles. This is a definition that seeks to acknowledge the wide variety of midwifery roles that are performed by employed midwives, and the pivotal, indeed "core", function that each of these has in relation to the continued day-to-day running of New Zealand's maternity facilities. The range of different jobs and functions that core midwives perform is considerable, underlining the diversity of roles that exist within core midwifery. The sustainability of the practice of core midwives is a significant issue for the future of sustainable maternity services nationally and internationally. 
There is a history of persistent core midwifery vacancies across New Zealand, affecting some DHBs in particular (Kyle \& Aileone, 2013). The main issues noted by the Midwifery Employee Representation and Advisory Service (MERAS, 2014) are distribution of the workforce and retention of midwives, particularly in some of the larger maternity units. According to the New Zealand College of Midwives (NZCOM), some maternity units in New Zealand's hospitals are in what is described as an emergency situation due to midwifery shortages (Williams, 2017). The Midwifery and Maternity Provider Organisation's (MMPO's) mapping project found that, between 2011 and 2013, the number of hospital midwives dropped from 1444 to 1345 . This was in part due to some midwives in rural areas choosing to become self-employed LMCs. Rural communities across the country are particularly vulnerable to shortages if a midwife retires or leaves the area, as it can take up to two years to recruit a replacement (Health Workforce New Zealand, 2015). Furthermore, there is a national shortage of midwives in a number of countries across the world and many countries face problems relating to attrition of the midwifery workforce (Hunter \& Warren, 2014; Pugh, Twigg, Martin, \& Rai, 2013; United Nations Population Fund, International Confederation of Midwives, \& World Health Organization, 2014).

The impact of shortages of midwives can lead to suboptimal staffing standards, potentially affecting the quality and safety of maternity services for women. MERAS has encouraged DHBs to "develop contingency plans to mitigate the risk to services and implement action plans towards meeting the staffing standards which would also assist in recruitment and retention of midwives" (MERAS, 2014, p.1). This study provides insights into conditions that support the sustainability and retention of core midwives and, therefore, holds the potential to significantly inform maternity service provision in New Zealand and internationally.

\section{Literature review}

Midwifery is one of those professions that can be physically, emotionally and mentally demanding, making midwives particularly prone to stress and burnout (Schaufeli \& Janczur, 1994; Young, Smythe, \& McAra-Couper, 2015). This applies to both caseloading midwives as well as core midwives; however, the kind of pressure each group is exposed to differs substantially (Yoshida \& Sandall, 2013). While caseloading midwives have been thought to suffer mostly from the personal and social constraints of constantly being on call (Donald, Smythe, \& McAra-Couper, 2014; Engel, 2003; McLardy, 2003; Todd, Farquhar, \& CamilleriFerrante, 1998; Young et al., 2015), for core midwives burnout has been linked to lower job autonomy and longer working hours (Mollart, Skinner, Newing, \& Foureur, 2013; Yoshida \& Sandall, 2013).

Other issues core midwives in particular have encountered include: role conflicts, harassment and bullying by colleagues and family of clients, and greater workload in relation to staffing levels (Yoshida $\&$ Sandall, 2013). Conflict with colleagues, such as medical doctors or between senior and junior midwives, is a recurring theme when investigating stressors for those midwives working in a hospital setting (Banovcinova \& Baskova, 2014; Hunter, 2004). Hunter $(2004,2005)$ proposed that at the centre of these work conflicts lies an ideological clash as midwives working in a hospital setting have to conflate the ideal of providing individualised womancentred care with the more technology-intensive, tightly protocolgoverned, institutional demands of the hospital. This clash in itself constitutes an internal conflict and can lead to disillusionment as midwives may feel they no longer can live up to their personal professional standards (Hunter, 2005).

Another source of stress core midwives contend with are pressures resulting from staff shortages (Curtis, Ball, \& Kirkham, 2006; Kirkham, Morgan, \& Davies, 2006). Such shortages result in midwives being expected to cover or do the same work with fewer people, which leads to the dissatisfaction of not having the time and resources to provide optimal care. Shift work has also been associated with an increase in work-related stress and burnout (Kirkham et al., 2006; Yoshida \& Sandall, 2013). In their survey of hospital and community midwives, Mollart et al. (2013) found that those midwives working a mix of day and night shifts had poor morale, which is one of the signs of burnout. Moreover, shift work has been linked to a range of health issues such as poor diet, insomnia or other sleep disorders, irritability and headaches (Zhao \& Turner, 2008), as well as an increase in risk for anxiety, hypertension, tiredness and stress (Di Milia, Waage, Pallesen, \& Bjorvatn, 2013; Flo et al., 2012). Consistent nightshifts in particular can heighten stress levels and impact family life (Presser, 2000).

All these issues have been found to negatively impact midwives' experiences of their job and ultimately may lead to a decision to leave practice, which can put a further strain on remaining staff (Curtis et al., 2006; Wakelin \& Skinner, 2007). While these findings seem to paint a fairly bleak picture of the profession, researchers have started to create a productive counterpoint by focusing their investigations on the factors that motivate and sustain midwives to continue with their work (Brodie, Warwick, Hastie, Smythe, \& Young, 2008; Davies, Daellenbach, \& Kensington, 2011; Engel, 2003; Gilkison et al., 2015; Kirkham et al., 2006; McAraCouper et al., 2014; McLardy, 2003; Sandall, 1997; Sullivan, Lock, \& Homer, 2011; Wakelin \& Skinner, 2007). The concept of sustainability refers to the ability of systems, processes or people to continue without exhausting their resources (Crowther et al., 2016). With regard to midwifery, studies exploring sustainability of practice have focused on factors that maintain midwives' passion and commitment to their profession and the concepts or interventions that support midwives to cope with the stresses of their job (Crowther et al., 2016).

Research from the international arena also suggests that relationships with women are an important part of what sustains midwives in any context. For example, research among National Health Service (NHS) midwives in the United Kingdom (UK) (Kirkham et al., 2006) and hospital midwives in Australia (Sullivan et al., 2011) showed that midwives working in these settings gain a great sense of satisfaction from their relationships with the women in their care. Being able to establish an effective relationship with women is fundamental for midwives to practise well, as these encounters, however short or fragmented they may be, can have a great impact on the woman's emotional state and her overall maternity experience (Halldorsdottir \& Karlsdottir, 1996).

Supportive relationships with colleagues and management, and the sense of community and belonging that comes with collegial relationships, also rank high among the factors that reduce midwives' stress level on the job and have been listed as reasons to keep practising (Kirkham et al., 2006; Sullivan et al., 2011; Yoshida \& Sandall, 2013). Hunter (2005) noted that for hospital midwives it was their colleagues rather than their clients who were the most important source of professional feedback.

Core midwives were also found to take great pride in their profession as they regarded it as a privilege to be able to be present during births (Sandall, 1997; Sullivan et al., 2011), which helped 
them deal with the stresses of their job. Others indicated they enjoyed the variety of roles their profession brings (Kirkham et al., 2006). For these midwives, the unpredictability and variability of their work and the large range of skills required are sources of excitement that keep them interested and engaged. Higher levels of occupational autonomy were also associated with reduced stress (Yoshida \& Sandall, 2013) and greater job satisfaction and morale (Kirkham, 2011).

The current study seeks to contribute to the accumulation of research into sustainability among core midwives, which is an issue that has not been explored in the New Zealand context previously. In seeking to identify factors and strategies that support core hospital midwives to carry out their practice in a durable way, this research is ideally situated not only to contribute to the growing body of work on sustainable midwifery but to the provision of effective, sustainable maternity services across New Zealand and internationally.

\section{METHOD AND METHODOLOGY}

Potential participants were invited to participate in this research via an email circulated to midwives through the NZCOM database. The email invited recipients, who would like to participate in the study or to find out more about the study, to email the project's research officer. For the 22 midwives who consented to participate, semi-structured interviews lasting approximately an hour were organised at a time and place of convenience to the interviewee, outside of their workplace. The interviews were carried out in person, by telephone or via Skype, depending on the location and preference of the research participant. The interviews were recorded, and participants were provided with an opportunity to review and make changes to the transcript of their interview, prior to it being included in the research analysis.

Data analysis was informed by a qualitative, descriptive approach (Braun \& Clarke, 2006). Interview transcripts were read by different members of the research team for emerging themes and patterns. On the basis of team discussions, a list of key themes, referred to within the team as "codes", were identified. Transcripts were then read and analysed using these codes, and interview data were allocated to individual codes with some data being identified as relevant to two or more codes. Team discussion was ongoing throughout this process in order to facilitate accurate and consistent coding. This also allowed for a small number of new research codes to emerge as discussion and reading of the data became more nuanced. Each code was subsequently analysed separately for sub-codes, and was then considered in relation to other data codes in order that overlaps, cross-overs and relationships could be identified. This enabled analysis of the patterns, both within and across the different codes, to occur.

The research received ethical approval from the Auckland University of Technology Ethics Committee (AUTEC reference number 14/380). The project was also reviewed by the Joan Donley Midwifery Research Collaboration (JDMRC) Database Access Governance Group, which agreed that the research met the criteria for access to the NZCOM membership database.

\section{Participants}

The 22 midwives who participated in this study came from a range of jobs and functions but all had worked between 8 and 40-plus years as core midwives. Ten of the midwives worked in tertiary level units, 5 in secondary level units, and 5 in primary units, with the remainder working in other employed capacities. Twelve research participants were based in the North Island of New Zealand and 10 in the South Island. Some of the midwives who participated in the study regularly rotated to different wards of a maternity unit; others worked almost exclusively on a particular ward. Some midwives worked in urban areas and others rurally; some were specialists in a particular field of midwifery; some were charge midwives or worked at another level of management; and a number had experience of working two midwifery jobs at the same time. The majority of midwives worked shifts, including rostered shifts over the weekend, although some did work fixed hours.

\section{FINDINGS}

\section{Core midwifery skills}

This research has found that the sustainability of core midwifery for our participants relies on having effective relationships with women, whānau, colleagues and managers. Along with relationships, these core midwives are sustained by the love of the job and the variety and diversity of work options that being a core midwife afford. For core midwives to feel sustained they also need to feel valued for the work they do and have control over their working conditions. This research has identified strategies that this sample of core midwives has developed to sustain themselves, such as ways that they look after themselves, and arrange their work and life. Core midwives who participated in this study spoke of feeling invisible and sometimes undervalued, but this research has identified the very special skills that core midwives have, and shows their unique contribution to New Zealand's maternity service.

It is the significant core midwifery skills that this paper explores. Other themes will be explored in forthcoming articles. From the interviews emerged an appreciation of the special and unique skills that core midwives have developed. Core midwives thrive on the diverse and varied nature of their roles. Midwives in this study spoke of quickly building a partnership with a woman whom they have probably never met, and who may have very complex needs. Core midwives need to be skilled and prepared to deal with everything, including the unexpected and critical incidents, while at the same time working in partnership with the women and guarding the normal as much as possible. The skill of managing a unit, creating the culture of that unit and supporting students, new graduates and LMCs, as well as the women being cared for in that facility, is another role that a core midwife can be asked to take on.

\section{Core skill of connecting with women quickly}

As a part of everyday practice, core midwives meet and care for women they are unlikely to have met previously, and often at a time when the women are in distress. Core midwives in this study speak of working with every woman who "comes through the door", and of the skill of being able to form a partnership with each woman.

One midwife puts it this way:

I think I'm quite good at that because I've been doing it for a long time and I am able to get a connection with the woman very, very quickly to make that a really good experience for her but at the same time to have a lot of professionalism. And also knowing that women need hospital midwives. I am happy to provide that care; I am happy to be the one who comes in and, you know, hopefully, makes it a good experience.

Midwife participants seem to retain the same strong philosophy of working in partnership with women alongside self-employed midwives who are able to work in partnership over the whole childbearing period for the woman. What is different is that their work with women is likely to encompass complicated and complex 
issues; however, the core midwife keeps her focus on the woman. One midwife talks about the needs of high risk women:

I think that for me women who have complications, who have problems during the pregnancy, deserve really good midwifery care just as much as the women who are normal and can birth at home.

It is not only physical complexity which this midwife sees; she is aware of multiple social complexities as well:

I see a lot of women who have terrible obstetric histories; who have all kinds of, not just physical health problems, but social stresses, a lot of socio-economic things. A lot of the time having a baby isn't the main thing they are thinking about in their pregnancy. They have a lot that they are juggling and I have always thought that those women need really good midwifery care too. So, for myself, in order to be able to provide that, I focused on learning to be confident with high risk, with the more medical interventions to do with pregnancy, which I know is not usually a standard midwifery philosophy. I enjoy helping women get positive experiences when it is that much more of a challenge for them to have a positive experience, if that makes sense.

For this core midwife it is not only the obstetric complexity, but the social complexity and diversity of women for whom she provides expert care; trying to make it into a positive experience for them in whatever ways she can, and also protecting normal birth in complex situations.

\section{The skill of guarding normal while in a technological world}

Some of the participants point to the fact that women have different needs and different views on what matters for them during childbirth. These core midwives provide individual care and adapt their philosophy to meet the needs of individual women and to work around the medicalised environment of the hospital setting. One midwife speaks of preserving the experience of women becoming mothers (which she describes as normalising) wherever possible, so that it is meaningful for the woman and always keeping the woman at the centre of care:

I try to normalise the woman's experience. It is not that I naively think that all birth is normal because I don't, but I think to make it as normal and meaningful for the woman and her family. That has always been my thing that has kept me going. So even in very abnormal situations to be able to do that and to place the woman at the centre of everything I do. That has always been my philosophy - that she is there, in the centre.

Despite working in an increasingly medicalised environment, core midwives in this study are very clear that the focus of their care is on the woman and the fact that she was becoming a mother, as well as providing midwifery care required for women experiencing complications. For them, the skills of core midwifery, therefore, encompass both the skill of caring for women with complex needs, as well as preserving the special event for the woman and her family of welcoming a new baby.

\section{Core midwifery skill of anticipating: Keeping women safe}

Another midwife explains her skill of anticipatory knowledge, which comes from experience, and also her ability to anticipate and know when things are not right:
Yeah, you get a sense quite early on, you know. You kind of have a picture in your head when you take over and it's probably experience as well because I've been working in hospitals for such a long time, I've got that kind of experience that you know 'Ooh, there are certain alarm bells that are going off and 'This is what I'm looking for, and I'm hoping this is the presentation I get'. But I also can anticipate and early on I can sense that something is not going right.

The ability to anticipate early and having an intuitive sense of what is happening when she first assesses a woman can trigger "alarm bells". For core midwives, a lack of previous knowledge of, or relationship with, a woman means that they need to rely on good assessment skills, highly developed intuition and early anticipation as means of supporting safety for the woman and her family.

\section{The range and diversity of core midwifery skills}

Some of the core midwives in this study work in complex and high stress secondary or tertiary environments. Some midwives love the diversity and enjoy the experience of emergencies because there is a sense that midwives are at a very significant event in people's lives, an event that won't be forgotten and it feels a privilege for midwives to be with women during such a special life event.

There are occasions when birth does not go to plan, but the core midwife helps:
But also, you know, in the times where something is not so nice and someone loses a baby - like - if you think what you did even just a little bit helped people through that horrible time - like - that kind of thing, that kind of sustains you because you know, at the end of the day, what you did wasn't just for money - like - it isn't just because you need to survive. Like you actually - maybe not a big difference - but just made a little bit of difference. Like that is quite important for me out of a job because you spend so much time doing it I think you want to have that kind of good feeling about it.

The role of the core midwife is to attend women when her risk status alters and there is transfer of care from her primary care midwife (LMC), when, for example, the baby has died. In such cases, this midwife says she makes sure she helps the woman and family by making a little bit of a difference. It makes her feel good to think she has helped. This midwife shows sincere empathy in her work with families experiencing loss and acknowledges how horrible the situation can be. Yet in this sadness, this midwife feels better by knowing she has done her job really well and has done it for the love of the job, not just money.

On the other end of the spectrum, in primary units, some core midwifery roles focus more on care related to physiological birth, breastfeeding and postpartum care. Midwives in these roles also emphasise the positive nature of that role. The following participant identifies the positive aspects of her role in this way:

I get to go to so many lovely physiological births. Truly people give birth, you know, by themselves. I get to go to so many lovely physiological births and it is so different looking after those women and their babies postnatally compared to [looking after] the babies that have come from the base hospital, you know, that have been pulled out with forceps, you know. They [the mothers] can't breastfeed because they've had an augmented labour and an OP position, you know, with masses of drugs ... and the poor women who feel like they've failed... So one of the things 
that I love about my job is doing nights sometimes, so going to lots of physiological births. And, sometimes, I get to help people debrief a bit from their really traumatic birth, you know, in the middle of the night, sitting there, [the woman] breastfeeding and talking about stuff.

Core midwives are sometimes able to attend normal births as second midwife, working together with the woman's LMC midwife - a situation that is common in smaller birthing units, rural and urban, in New Zealand. This midwife speaks of the variety of her roles in attending many normal births, debriefing with women who have had traumatic births, helping with breastfeeding and listening and talking with clients in something of a therapeutic capacity. For the midwives in this study, working with women after the birth provides immense satisfaction, an example of when working on the night shift with mothers and babies can be particularly fulfilling. Whilst core midwives have limited opportunity to provide continuity of care, it is possible, from this one midwife's testimony, that many value working with women and other midwives to support normal birth and provide help and advice for women when caring for their newborn babies.

\section{The skill of complexity and diversity}

Part and parcel of being a core midwife is being skilled in caring for complex women and skilled with emergencies. Many midwives in this study are very proud of their ability and skills in this area.

One midwife talks about the skill of being prepared for potential problems that she has developed as a core midwife:

\section{It's about thinking actually that [it] is also [possibly] not normal. It's about thinking just a little bit more about, you know, have you got everything ready for an emergency? You know, what happens if there's a potential problem? I think it is really important to do the PROMPT [PRactical Obstetric Multi-Professional Training] course just to keep yourself updated with emergency stuff because when it happens, it happens really quickly. You are expected to be able to know what to do in an emergency, even to lead the emergency and, unfortunately, we see too many of them. But that is also the nature of our clients. They can be really, really unwell.}

For a core midwife who works in a primary unit, dealing with emergencies is also something which adds to the variety of her role. In the primary setting with the tertiary unit being quite some distance away, another layer is added to the complexity. Those of our participants who work in primary units speak of the range of emergencies situations they have encountered in those environments, and of the ways in which they have worked with, and supported, clients through those emergencies.

\section{In the primary setting with the tertiary unit being quite some distance away, another layer is added to the complexity.}

They speak of the reality of providing such care in primary settings that may be a considerable distance from a tertiary or secondary unit; of the skills and readiness required; of the necessity for team work, and of the difficulties of the assumption that's made that core midwifery in a primary unit is "stressless". In the words of a core midwife in a primary unit: "So it actually wasn't that stressless". She added, however, "It was really good". This incredibly challenging, diverse and complex nature of core midwifery is part of what some midwives in this study say sustains them.

\section{The skill of being prepared for everything, including the unexpected}

One of the factors that supports sustainability is the variability and unpredictability of their work. This unpredictability means that they often don't know what is going to "come through the door next". Whether the participants work in a small primary unit or in a secondary/tertiary unit, they testify to core midwives becoming very skilled at being prepared for everything. As one midwife says:
I think one of the things that keeps me going for so long is no two days are the same. Midwifery is a very humbling kind of career because every time you get cocky and think you know it all, someone throws a curve ball and - you know - shocks you. So I find that really enjoyable. I never know what I'm [going to be] doing from day to day. It changes and you can be sitting on a shift thinking 'oh, this is all quiet...' and something comes through the door which completely throws you off kilter.

The core midwives are aware that in their job they can never get overconfident, or think they know it all, as they need to be always ready to respond to the unpredictable nature of the job, especially when working in a hospital setting. To be able to deal with such a dynamic work environment is a real skill and takes mental preparation. As one midwife says:

I quite like the dynamic nature of the workplace. I am a
person who doesn't really like every day to be the same, you
know. I think I would struggle with a 9 to 5, I really would.
I do like going to work and not knowing who I am going
to work with and not knowing what is going to happen in
a day and I like the challenge, it really challenges me to do
this work in so many ways. And I really like that it does
that. I like having to be mentally prepared. I like having to
be, you know, be in a good space to do the job and I like to
get the best from who I work with, - you know - all those
kinds of things, and I guess I like the challenge.

One core midwife who works in a rural unit describes herself in the following way:

I work in a unit in the country, and it does mean that our midwives really need to be 'Jills of all trades'.

A "Jill of all trades" sums up the nature of core midwives who work in primary units, often in rural and remote areas. This description suggests the need for primary unit core midwives to have a variety of knowledge and practical skills (sometimes unrelated to midwifery) so that they can provide quality midwifery care.

\section{The core skill of managing a unit}

Usually each area on each shift has a charge midwife who oversees what is happening in the unit. It would be the more experienced core midwives who would step into this role.

[When you are] the person being in charge because it is not just one person - you are [not] advocating for one person; you are really focused on... it is your responsibility to make sure that everyone is doing ok. So, all of the patients and their families and all of the midwives and the health care assistants and the doctors and everyone you are working with, as part of a team...you have to have this kind of helicopter view of everything that is going on. 
As the midwife in charge, this core midwife has developed the skill of having a "helicopter view". To manage a unit in such a way requires considerable expertise, in particular in communication skills, so that everyone from the consultant to the health care assistant feels appreciated and valued, and good communication lines are established.

In the opinion of our participants, core midwives sustain and are sustained by being a part of a maternity unit that supports and nurtures students, new graduates and all staff who come into the unit. The participants refer to this as a "nest". Core midwives, they indicate, work hard to create a learning culture within their environments which makes the facility a safe place for people to ask for help and advice. Ultimately a learning culture will impact positively on the care of mothers and babies, which is an important priority for midwives.

\section{Core midwives create a "nest"}

One of the aspects of creating the culture of the unit is the way core midwives foster and support students and new graduate midwives. Core midwives in this study speak of the way that they feel the maternity facility creates a "nest" for students, new midwives and new mothers. One midwife, who was interviewed in her garden where a bird was nesting in the tree above her, likens this to a mother bird and her chicks:

\section{I've got a bird's nest above me and I would think a mother bird comes and she feeds those chicks up there and I mean she is nurturing those chicks to grow up in the world and have their own children and do exactly what she is doing, you know. It's nurturing, it's nurturing the new students, it's nurturing the new midwives, it's nurturing the mothers and babies.}

Likening experienced midwives in the facility to a mother bird gives a sense of them as nurturers who know when the new students, new midwives and new mothers are ready to fly and leave the nest and be independent. There is quite a skill to working alongside a new graduate or a student, giving them the opportunities to practise in a safe way, and gradually increase their confidence. Another midwife talks about passing on self-care skills to students, as well:

When the students are here and they are working with
someone I'm always saying to them 'Are you looking after
yourself?' I would say, 'You are entitled to have your
breaks'. ... And even now the midwives who work in the
ward making sure they do get a break, you know. If they
are going to be there for 12 or so hours they have got to be
able to look after themselves first so they can care for the
women as well.

In this way, the core midwife is passing on a sustainable way of working to student midwives by ensuring that they look after themselves and have breaks.

\section{The skill of flexibility and adaptability}

The roles that core midwives perform are diverse and varied, from day to day and from position to position. Core midwives in this study developed the skill of flexibility and adaptability in order to flourish, and value the variety in their work as a way of sustaining themselves in core practice. They speak about changing their midwifery roles so that work fitted better with their life outside of work and also sometimes to prevent them from becoming what some described as "stale", or of moving jobs and wards to help get through difficult times. In the words of one participant: "diversity is my sustainability".
For me I have to keep moving. I have to keep things fresh. I get bored. I've got a lot of energy and I like to keep moving and I don't want to get stagnant sitting in the same department for 10 years doing the same job.

Midwives who have, or have had, more than one midwifery role speak of finding that the work they do in a particular job might feed into and support their work in another field of midwifery; thereby bolstering and supporting them in both areas of their practice. For other midwives, one of the benefits of midwifery being diverse is that it also enables them to focus upon becoming skilled in a particular area of work that they enjoy. In such instances it is seen to be partly their love and familiarity of that area that sustains them.

\section{I've actually always concentrated on postnatal. I'm a real postnatal midwife, because I find the delivery unit just doesn't give me the excitement that other midwives get.}

Indeed, whilst the variety offered by core midwifery is seen to be important to many midwives, it is also acknowledged that there is a limit to the variety that employees can enjoy or manage well. In the words of one midwife:
Management often talk about flexibility or they used to. It was flexibility before resilience, but you just get this impression of this ideal employee or midwife who has an ability to literally bend over backwards and to go wherever you send them. That's another thing that they think is important, you know. A midwife to cope with the unpredictability of obstetrics and the flow, the ideal midwife will rotate around all the areas, so that they visualise us, I'm quite sure - really - as just... we are just numbers and we are ideal if we can at the drop of a hat go into the tertiary or primary facility and give them a hand when they are busy and rotate from maternity to delivery suite.

While the skills of being flexible and dealing with the unpredictable nature of obstetrics are sustaining, there is a limit. If management sees core midwives purely as entities who can be moved from area to area and cope with whatever they face, then this will lead to dissatisfaction. Participants suggest that the moving/rotation/ variety needs to be determined in large part by the midwives themselves, rather than enforced by management.

\section{Valuing and making visible}

Perhaps sometimes core midwifery is invisible, and the complex skills and decision making, which core midwives do every day, can go unnoticed amidst a plethora of more task-oriented duties. But as one midwife says:

$$
\begin{aligned}
& \text { Actually there is a lot of midwifery skill involved in being a } \\
& \text { core midwife and it's not just running pumps and looking } \\
& \text { after epidurals. }
\end{aligned}
$$

\section{Not "just" a core midwife}

Despite the incredible skills which these core midwives have spoken of in this study, and the diversity of the work they carry out, they frequently do not give enough credit to their own role and value. Nor do they necessarily feel that others do. This midwife describes the importance of valuing herself in her role:
It's so important because I meet so many midwives and they say 'I'm just a core midwife' and occasionally I've heard myself say it too, I'm just a... But I don't think I actually say it, I say 'just' a lot, but I never call myself 'just a core midwife' because I'm really proud to be a hospital midwife. 
I'm very proud to be a midwife, actually. I don't see myself as any particular role; I don't put a title on myself; I see myself as a midwife. I happen to work in a hospital and sometimes I go out somewhere else and sometimes I do something else.

This midwife emphasises the need for midwives themselves to identify their value as well as the importance of being valued by others, regardless of their specific midwifery role. She discards specific titles and identifies herself as a midwife who works in various places but it is the midwifing that is the thing to be really proud of. She tries to influence colleagues to see themselves as important assets and suggests they are not "just a core midwife".

\section{DISCUSSION}

In answering the question "what sustains core midwives in New Zealand?", participants in this study provide a myriad of data which reveals what they feel is at the heart, or the core, of core midwifery. The metaphor of a "Jill of all trades", used by one participant, may well encapsulate the skills which core midwives have developed. Our findings suggest that core midwives juggle the varying needs of women and babies within the multiple contexts of maternity facilities across New Zealand.

The ability to connect and form a partnership relationship with women very quickly, often in times of complexity, uncovered in this study, resonates with the findings of others who have explored the sustainability of core midwives (Hunter, 2005; Kirkham, 2011; Sandall, 1997; Sullivan et al., 2011; Wakelin \& Skinner, 2007). Our study reinforces the description that core midwives provide care for women and their whānau in often very complex situations, yet they keep the woman at the centre of care and strive to preserve a normal as possible experience for her. The findings of this study concur with others such as Sullivan et al. (2011), who found that Australian midwives gained satisfaction from working with birthing women and this is what held them in practice. Relationships are equally as important for midwives in this study, and it is the particular skill of building that partnership relationship quickly, often in a complex situation, which core midwives in this study describe as integral to good core practice. Along with this, core midwives in this study speak of the skill of guarding the normal experience as much as possible while the woman may require complex care. Core midwives in this study speak of seeing the woman as a woman becoming a mother, not just as "the diabetic in room 2".

\section{The metaphor of a "Jill of all trades", ... may well encapsulate the skills which core midwives have developed. Our findings suggest that core midwives juggle the varying needs of women and babies within the multiple contexts of maternity facilities across New Zealand.}

Some of the very things described as helping to sustain core midwives in this study could also, at other times, be a source of stress. Whereas Mollart et al. (2013) found that complexity and diversity of women could contribute to burnout for Australian midwives, in this study a number of participants find aspects of enjoyment in just that complexity and diversity. Indeed, some midwives we spoke with suggest that it is in part the unpredictability and variety of being a core midwife that sustains them and stops them from feeling stagnant in their work. Furthermore, core midwives need the skills of flexibility and adaptability due to the diverse and varied nature of their roles. However, research participants point out that there are limits to the flexibility and adaptability that can be expected of midwives, as there are for any employees. These midwives speak of needing to have choice in relation to the areas they work in and the ways in which they work, rather than simply being moved around at the will of maternity management in order to plug gaps in services. There is a balance to be achieved between core midwives themselves making choices about the areas where they work, and being used simply as an entity to staff maternity facilities. In the former, midwives have the control and choice, and this is what sustains them. If that control over the way they work is taken away, then that may lead to an unsustainable working environment.

\section{Core midwives sometimes feel they are invisible and undervalued for the important role they play in the New Zealand maternity services; yet, from the rich data examined intensively in this research, we can argue it is the core midwives who are fundamental to the effective functioning of New Zealand maternity services.}

A further skill which some senior core midwives develop is that of taking on a leadership role within a maternity facility and, as leaders, they have a significant impact on the culture of the unit. In their role of always being present in maternity units, it is the core midwives who support students, new graduates and all staff who come into that unit. It is the core midwives who manage the unit and create the culture of the unit.

Core midwives sometimes feel they are invisible and undervalued for the important role they play in the New Zealand maternity services; yet, from the rich data examined intensively in this research, we can argue it is the core midwives who are fundamental to the effective functioning of New Zealand maternity services.

\section{Strengths and weaknesses of the study}

This study is based upon in-depth qualitative interviews and has therefore generated rich data on the research participants' experiences of their working environments and of the ways in which they perceive and understand their sustainability as core midwives in New Zealand. The study involved descriptive analysis of the interview narratives of 22 core midwives working in diverse settings and geographical areas of New Zealand. The findings of this research are not generalisable to all core midwives; yet they provide valuable insights into the types of issues around sustainable practice that core midwives in New Zealand appear to be currently encountering.

\section{CONCLUSION}

Whether the woman is experiencing a normal birth at a small rural unit or a complex birth in a large urban tertiary unit, there is likely to be a core midwife involved in some way. Often invisible, the core midwife is the backbone of New Zealand maternity services as they are always "there", always prepared to care for whoever 
walks through the door. The findings of this New Zealand study, which explores the sustainability of core midwifery, resonates with previous studies and also take them further by identifying the diverse skill set which core midwives have honed, such as the ability to develop trust and empathy with diverse women in a very short time; the capacity to respond competently to a range of complex and emergency situations; and, importantly, the ability to create and sustain a supportive culture for the benefit of women, as well as both new and experienced staff.

\section{CONFLICT OF INTEREST STATEMENT}

The authors declare that there are no conflicts of interest.

\section{REFERENCES}

Banovcinova, L., \& Baskova, M. (2014). Sources of Work-related Stress and their Effect on Burnout in Midwifery. Procedia-Social and Behavioral Sciences, 132, 248-254. http://dx.doi.org/10.1016/j. sbspro.2014.04.306

Braun, V., \& Clarke, V. (2006). Using thematic analysis in psychology. Qualitative Research in Psychology, 3(2), 77-101. http://dx.doi. org/10.1191/1478088706qp063oa

Brodie, P., Warwick, C., Hastie, C., Smythe, L., \& Young, C. M. (2008). Sustaining midwifery continuity of care: Perspectives for managers. In C. Homer, P. Brodie, \& N. Leap (Eds.), Midwifery continuity of care: A practical guide (pp. 149-164). Sydney, Australia: Churchill Livingston.

Crowther, S., Hunter, B., McAra-Couper, J., Warren, L., Gilkison, A., Hunter, M., ... Kirkham, M. (2016). Sustainability and resilience in midwifery: A discussion paper. Midwifery, 40, 40-48. http://dx.doi. org/10.1016/j.midw.2016.06.005

Curtis, P., Ball, L., \& Kirkham, M. (2006). Why do midwives leave? (Not) being the kind of midwife you want to be. British Journal of Midwifery, 14(1), 27.

Davies, L., Daellenbach, R., \& Kensington, M. (Eds.). (2011). Sustainability, Midwifery and Birth. Oxon, United States: Routledge.

Di Milia, L., Waage, S., Pallesen, S., \& Bjorvatn, B. (2013). Shift work disorder in a random population sample-prevalence and comorbidities. PLoS One, 8(1), e55306. http://dx.doi.org/10.1371/journal.pone.0055306

Donald, H., Smythe, L., \& McAra-Couper, J. (2014). Creating a better work-life balance. New Zealand College of Midwives Journal, 49, 5-10.

Engel, C. (2003). Towards a sustainable model of midwifery practice in a continuity of carer setting: the experience of New Zealand midwives. New Zealand College of Midwives Journal, 28, 12-15.

Flo, E., Pallesen, S., Mageroy, N., Moen, B. E., Gronli, J., Hilde Nordhus, I., \& Biorvatn, B. (2012). Shift work disorder in nursesassessment, prevalence and related health problems. PLoS One, 7(4), e33981. http://dx.doi.org/10.1371/journal.pone.0033981

Gilkison, A., McAra-Couper, J., Gunn, J., Crowther, S., Hunter, M., Macgregor, D., \& Hotchin, C. (2015). Midwifery practice arrangements which sustain caseloading Lead Maternity Carer midwives in New Zealand. New Zealand College of Midwives Journal, 51, 11-16. http:// dx.doi.org/10.12784/nzcomjnl51.2015.2.11-16

Halldorsdottir, S., \& Karlsdottir, S. I. (1996). Empowerment or discouragement: women's experience of caring and uncaring encounters during childbirth. Health Care for Women International, 17(4), 361-379.

Health Workforce New Zealand. (2015). Health of the health workforce 2015. Wellington, New Zealand: Ministry of Health.

Hunter, B. (2004). Conflicting ideologies as a source of emotion work in midwifery. Midwifery, 20(3), 261-272. http://dx.doi.org/10.1016/j. midw.2003.12.004

Hunter, B. (2005). Emotion work and boundary maintenance in hospital-based midwifery. Midwifery, 21(3), 253-266. http://dx.doi org/10.1016/j.midw.2004.12.007

Hunter, B., \& Warren, L. (2014). Midwives' experiences of workplace resilience. Midwifery, 30(8), 926-934. http://dx.doi.org/10.1016/j. midw.2014.03.010

Kirkham, M. (2011). Sustained by joy: The potential of flow experience for midwives and mothers. In L. Davies, R. Daellenbach, \& M. Kensington (Eds.), Sustainability, Midwifery and Birth. Oxon, United States: Routledge.
Kirkham, M., Morgan, R., \& Davies, C. (2006). Why do midwives stay? Sheffield, United Kingdom: University of Sheffield.

Kyle, M., \& Aileone, L. (2013). Mapping the rural midwifery workforce in New Zealand. Wellington, New Zealand: Health Workforce New Zealand.

McAra-Couper, J., Gilkison, A., Crowther, S., Hunter, M., Hotchin, C., \& Gunn, J. (2014). Partnership and reciprocity with women sustain Lead Maternity Carer midwives in practice. New Zealand College of Midwives Journal, 49, 29-33.

McLardy, E. (2003). On-call 24/7: Midwives negotiating home and work boundaries (master's thesis). Victoria University, Wellington, New Zealand.

Midwifery Council of New Zealand. (2015). 2015 Midwifery workforce survey. Wellington, New Zealand: Author. Retrieved from https:// www.midwiferycouncil.health.nz/images/stories/pdf/Publications/ WorkforceSurvey2015_150.pdf

Midwifery Employee Representation and Advisory Service. (2014). Midwifery staffing standards. Christchurch, New Zealand: MERAS.

Mollart, L., Skinner, V., Newing, C., \& Foureur, M. (2013). Factors that may influence midwives work-related stress and burnout. Women and Birth, 26(1), 26-32. http://dx.doi.org/10.1016/j. wombi.2011.08.002

Presser, H. B. (2000). Nonstandard work schedules and marital instability. Journal of Marriage and the Family, 62(1), 93-110.

Pugh, J. D., Twigg, D. E., Martin, T. L., \& Rai, T. (2013). Western Australia facing critical losses in its midwifery workforce: A survey of midwives' intentions. Midwifery, 29(5), 497-505. http://dx.doi. org/10.1016/j.midw.2012.04.006

Sandall, J. (1997). Midwives' burnout and continuity of care. British Journal of Midwifery, 5(2), 106-111.

Schaufeli, W. B., \& Janczur, B. (1994). Burnout among nurses - a Polish-Dutch comparison. Journal of Cross-Cultural Psychology, 25(1), 95-113.

Sullivan, K., Lock, L., \& Homer, C. S. (2011). Factors that contribute to midwives staying in midwifery: a study in one area health service in New South Wales, Australia. Midwifery, 27(3), 331-335. http://dx.doi. org/10.1016/j.midw.2011.01.007

Todd, C. J., Farquhar, M. C., \& Camilleri-Ferrante, C. (1998). Team midwifery: The views and job satisfaction of midwives. Midwifery, 14(4), 214-224. http://dx.doi.org/10.1016/S0266-6138(98)90093-6

United Nations Population Fund, International Confederation of Midwives, \& World Health Organization. (2014). The state of the world's midwifery. USA. Retrieved from http://www.unfpa.org/sowmy

Wakelin, K., \& Skinner, J. (2007). Staying or leaving: a telephone survey of midwives, exploring the sustainability of practice as lead maternity carers in one urban region of New Zealand. New Zealand College of Midwives Journal, 37, 10-14.

Williams, K. (2017, March 20). Midwife shortage at major hospitals 'emergency situation'. Stuff. Retrieved from http://www.stuff.co.nz/ national/health/90615414/midwife-shortage-at-major-hospitalsemergency-situation

Yoshida, Y., \& Sandall, J. (2013). Occupational burnout and work factors in community and hospital midwives: a survey analysis. Midwifery, 29(8), 921-926. http://dx.doi.org/10.1016/j. midw.2012.11.002

Young, C. M., Smythe, L., \& McAra-Couper, J. (2015). Burnout: Lessons From the Lived Experience of Case Loading Midwives. International Journal of Childbirth, 5(3), 154-165.

Zhao, I., \& Turner, C. (2008). The impact of shift work on people's daily health habits and adverse health outcomes. Australian Journal of Advanced Nursing (Online), 25(3), 8-12,17-22.

\section{Accepted for publication June 2017}

Gilkison, A., McAra-Couper, J., Fielder, A., Hunter, M., \& Austin, D. (2017). The core of the core: What is at the heart of hospital core midwifery practice in New Zealand? New Zealand College of Midwives Journal, 53, 30-37.

http://dx.doi.org/10.12784/nzcomjnl53.2017.4.30-37 